\title{
Missing markers when estimating quantitative trait loci using regression mapping
}

\author{
OCTAVIO MARTÍNEZ† \& ROBERT N. CURNOW* \\ Department of Applied Statistics, University of Reading, Harry Pitt Building, Whitenights Road, P.O. Box 240, Reading \\ RG6 2FN, U.K.
}

\begin{abstract}
When using molecular markers to estimate the locations and sizes of effects of QTLs in plant populations a common problem is the loss of marker genotypes for many individuals. Here we present a method that uses the information from individuals with missing marker genotypes when fitting regression mapping models using two or three neighbouring markers. The approach uses other nearby markers to recover information from the individuals with missing markers. The method is presented in detail for the two markers regression mapping technique applied to backcross or double haploid populations. The method is exemplified with a simulated data set and with data on a quantitative character in double haploid lines of barley. Generalizations of the method to three markers regression mapping models and to $F_{2}$ populations are outlined.
\end{abstract}

Keywords: interval mapping, missing markers, molecular markers, QTL, regression mapping.

\section{Introduction}

The use of molecular markers in segregating populations of plants permits the estimation of the number, positions and sizes of effects of polygenes affecting quantitative characters. The positions are now referred to as quantitative trait loci (QTLs). (Here we use the acronym QTL for the singular: quantitative trait locus and QTLs for the plural: quantitative trait loci.) The estimation methods are based on the information supplied by the molecular markers given that we know the parent of origin of each marker allele. To analyse the data, different genetical hypotheses can be proposed about the presence of one or more QTLs at different positions in the chromosomes of the individual plants. These hypotheses can then be translated into statistical models and the plausibility of each model can be tested. This general concept has been used in a number of different estimation procedures (see for example: Weller, 1986, 1987; Lander \& Botstein, 1989; Luo \& Kearsey, 1989, 1991; Simpson, 1989; Knapp et al., 1990; Carbonell et al., 1992; Jansen, 1992).

\footnotetext{
*Correspondence.

$\dagger$ Present address: UASLP, Facultad de Ciencias, Iturbide 965, cp 78000 , San Luis Potosi, SLP, Mexico.
}

For several reasons, observations of some of the molecular marker genotypes in various individuals can be lost during the laboratory process. The proportion of missing marker genotypes can sometimes be very high. If a marker genotype is missing in a particular individual then, without a special approach, we will not normally be able to use the phenotypic information on that individual in the analysis. This will decrease the sample size with a consequent loss of statistical power. In this paper we present a method that uses neighbouring markers to recover partially the information that has been lost on a marker. The method is based on the use of regression mapping models (Haley \& Knott, 1992; Martínez \& Curnow, 1992).

Firstly, we present a brief explanation of the estimation of QTLs using the regression mapping approach. Secondly, we propose a method to solve the problem of missing markers, exemplifying our approach with a set of simulated data and with data from a quantitative character in a population of double haploid lines of barley.

\section{Regression mapping}

Regression mapping consists of regressing the phenotype of the individuals in a segregating population on the probability of one or more QTLs segregating at particular positions between the markers, given that we 
know the marker genotype of each individual, say

$$
Y_{i j}=\beta_{0}+\left\{\sum_{r=1}^{r=q} \beta_{r} P\left[Q_{r} \mid M_{j}, \theta\right]\right\}+\varepsilon_{i j},
$$

where $Y_{i j}$ is the value of the quantitative character in the $i$ th individual of the $j$ th marker genotype; $i=1,2, \ldots$ $n_{\mathrm{j}}, j=1,2, \ldots k$. The number of marker classes, $k$, depends on the number of markers that we are taking into account in the model, as well as the type of segregating population, backcross or $\mathrm{F}_{2}$, involved. In eqn (1), $P\left[Q_{r} \mid M_{j}, \theta\right], r=1,2, \ldots q ; j=1,2, \ldots k$, are the probabilities of a specific QTL genotype, $Q_{r}$, given the marker genotype of the individual, $M_{j}$, and the position of the QTLs, denoted by $\theta$. This is defined for $j=1,2$, $\ldots k$ and $r=1,2, \ldots q$, where the total number of different QTL genotypes, $q$, depends on the number of QTLs assumed by the model as well as on the kind of segregating population being analysed. The values of $\beta_{r}, r=1,2, \ldots q$, denote the effects of each one of the QTL genotypes and $\beta_{0}$ represents a baseline from which the QTL effects are measured. In eqn 1 we assume that the positions of the markers along the chromosomes are known with certainty. Of course this assumption is never fulfilled and thus the positions must first be estimated. The functions $P\left[Q, \mid M_{j}, \theta\right]$ in eqn 1 are known, except for the value of $\theta$. The term $\varepsilon_{i j}$ in eqn 1 represents the variation that is not taken into account through the other terms in the model. If the underlying environmental error has a normal distribution with mean zero and variance $\sigma_{e}^{2}$, then $\varepsilon_{i j}$ in eqn 1 has a distribution that is a mixture of normal distributions. This is because plants with the same marker genotype may have different QTL genotypes.

In the simplest case we use a pair of successive molecular markers, assuming that a single QTL is segregating between them. Given that we do not know the position of the QTL, $\theta$, the estimation procedure consists of fitting eqn 1 by least squares for a grid of values of putative positions for the QTL, say $\theta=t$, $0 \leq t \leq \delta$, where $\delta$ is the distance between the markers, expressed as the probability of recombination between them. Then we select as estimator for the position of the QTL the value of $t$ that minimizes the residual sum of squares of the model, RSS $(t)$, say $t_{\text {min }}$. In general, if $q$ QTLs are segregating then $\boldsymbol{\theta}$ is a vector with $q$ components and the search must be performed in the multivariate space $\left\{0 \leq t_{1} \leq \delta_{1}, 0 \leq t_{2} \leq \delta_{2}, \ldots 0 \leq t_{q} \leq \delta_{q}\right\}$, to find $\mathbf{t}_{\min }=\left(t_{1 \text { min }}, t_{2 \text { min }}, \ldots t_{q \text { min }}\right)$ such that $\operatorname{RSS}\left(\mathbf{t}_{\min }\right) \leq \operatorname{RSS}(\mathbf{t})$ for all other values of $t$. Thus the estimates of the positions of the QTLs are given by $\mathbf{t}_{\text {min }}$ and the estimates of the QTLs' effects are given by the least squares estimators of $\beta_{1}, \beta_{2}, \ldots \beta_{q}$ obtained when fitting eqn 1 with $\boldsymbol{\theta}=\mathbf{t}_{\min }$, say $\hat{\beta}_{1}\left(\mathbf{t}_{\min }\right), \hat{\beta}_{2}\left(\mathbf{t}_{\min }\right), \ldots$, $\hat{\beta}_{q}\left(\mathbf{t}_{\text {min }}\right)$. Models of this type can be fitted, in turn, to different sets of markers and the conclusions reached from the various analyses interpreted to provide estimates of the number, positions and sizes of effects of the QTLs.

This procedure had been presented by Haley \& Knott (1992) for $F_{2}$ populations using two or three markers at a time and assuming one or two QTLs and by Martínez \& Curnow (1992) for backcross populations under similar conditions. In these papers it has been shown that, when using pairs of markers, the method gives results similar to the Interval Mapping approach (Lander \& Botstein, 1989) but regression mapping has the advantage of being easy to generalize to use the information from more than one pair of markers at the same time. The simultaneous use of three markers allows the elimination of the ghost effects of QTLs in neighbouring regions and thus enables discrimination between the presence of one or two QTLs.

Because of the simplicity of the least squares approach in regression mapping, other factors, for example experimental design structure and locality effects, can be easily introduced into the model and estimated at the same time as the parameters of the QTLs.

\section{Missing markers}

To present the method that allows the use of information from individuals with missing markers we shall consider the backcross:

$M_{1} M_{2} \ldots M_{k} / m_{1} m_{2} \ldots m_{k} \times m_{1} m_{2} \ldots m_{k} / m_{1} m_{2} \ldots m_{k}$,

where $M_{g}$ and $m_{g} g=1,2, \ldots k$ are the two alleles that can be present at marker locus $g$. Assume that we have a map of the $k$ marker loci and thus we can calculate the recombination probability between each pair of loci. Denote by $\delta_{g}$ the recombination probability between the loci $g$ and $g+1, g=1,2, \ldots k-1$ and by $T$ the distance in centiMorgans of a location in the chromosome measured from the first marker locus, $g=1$. As we shall be allowing no interference, the distance will be measured in Haldane centiMorgans and we denote by $H()$ the Haldane mapping function relating a distance $T$ with a recombination probability, say $T=H(t)=-\frac{1}{2} \log _{\mathrm{e}}(1-2 t)$.

In looking for putative QTLs along a chromosome we can fit to each successive interval $g=1,2, \ldots k-1$ the regression model

$Y_{i g j}=\beta_{0}+\beta_{1} \Gamma_{g j}\left(t_{g}\right)+e_{i j}$,

where $Y_{i g i}$ denotes the value of the quantitative character in the $i$ th individual that, with respect to the flanking 
markers $g$ and $g+1$, is classified in one of the $j$ th marker classes; $j=1,2,3$ or 4 . The marker genotypes can be represented by the marker gametes from the $\mathrm{F}_{1}$ parent, $M_{g} M_{g+1}, M_{g} m_{g+1}, m_{g} M_{g+1}$ and $m_{g} m_{g+1}$. We define $j=1$ if the genotype of the individual is $M_{g} M_{g+1}, j=2$ for $M_{g} m_{g+1}, j=3$ for $m_{g} M_{g+1}$ and $j=4$ for $m_{g} m_{g+1}$. In eqn $2, \Gamma_{g j}\left(t_{g}\right)=P[A \mid$ marker genotype $j$ with respect to the markers $g, g+1 ; t_{g}$ ] is the probability of having a QTL allele ' $A$ ' from the $\mathrm{F}_{1}$ parent, given the marker genotype of the individual with respect to the markers $g$ and $g+1$ and a putative position for the QTL within the interval in terms of recombination probability $t_{g}$ where $0 \leq t_{g} \leq \delta_{g}$. The form of these functions depends on the degree of interference that is assumed to be present in the species studied. In the case of no interference:

$$
\begin{aligned}
& \Gamma_{g 1}(t)=1-\frac{t\left(\delta_{g}-t\right)}{\left(1-\delta_{g}\right)(1-2 t)}, \quad \Gamma_{g 4}(t)=1-\Gamma_{g 1}(t), \\
& \Gamma_{g 2}(t)=\frac{(1-t)\left(\delta_{g}-t\right)}{\delta_{g}(1-2 t)}, \quad \Gamma_{g 3}(t)=1-\Gamma_{g 2}(t) .
\end{aligned}
$$

Having fitted model 2 to a grid of values of $t_{g}$ in each interval, $g=1,2, \ldots k-1$, we can graph the residual sum of squares of the model, RSS $\left(t_{g}\right)$, looking for evidence of a segregating QTL in one or more of the intervals (Martínez \& Curnow, 1992). If in the data set all the individuals have known marker genotypes at the markers $g$ and $g+1$, then all the individuals can be used to estimate the parameters of eqn 2 . In many cases the information about one or both of these markers may be missing and all of these observations omitted from the estimation procedure, with a consequent loss of information. If different individuals have different missing markers the model for different intervals will be applied to different sets of data, making it difficult to compare the results of the model applied to one interval with the results of the model applied to neighbouring intervals. If there are no missing data, the graph of the RSS $(t)$, against $t$, the position of the putative QTL, is continuous except for small random gaps at the positions of the markers. These small gaps are due to the arbitrary division of the plants into four classes rather than the two classes when carrying out the analysis at any marker position $g$, with the division differing between the intervals $\{g-1$ to $g\}$ and $\{g$ to $g+1\}$. The correct value of the RSS at the marker positions will lie within the small gaps and therefore not justify special calculation. When marker information is missing the graph of $\operatorname{RSS}\left(t_{g}\right)$ will have larger gaps at the corresponding marker positions, reflecting the fact that different sets of data have been used to fit the model. If we discard all the observations where one or more markers are missing, few observations may remain and the power of the analysis will be greatly diminished. The correct solution to this problem is, for each position in the chromosome, to use the information from the nearest available markers flanking that position in each particular individual. Thus for each position on the chromosome we calculate for each individual the probability of a QTL allele from the $F_{1}$ parent using the nearest flanking marker or markers. Information from more distant markers is of course irrelevant. The following two alternatives cover all the possibilities where it is possible to recover some information. (i) For some, but not all individuals, there are no markers present on one side of the position being studied, $T$. Then we can use the one known marker genotype to calculate the probability of having a QTL allele from the $F_{1}$ parent at the position $T$. To generalize we denote by $u$ the known marker and denote by $t_{u}$ the recombination probability between the position $u$ and the putative position of the QTL, $T$. (ii) The nearest known flanking markers are at positions $u$ and $v$, which may or may not be the marker loci $g$ and $g+1$ when $T$ is in that interval. Denote by $\delta_{u}$ the recombination probability between the marker loci $u$ and $v$, and again by $t_{u}$ the recombination between the marker $u$ and the putative position of the QTL, denoted by $T$. Now for computing purposes we can redefine the regression mapping model as:

$Y_{i}=\beta_{0}+\beta_{1}\left(\Gamma_{u}\left(t_{u}\right)+\Gamma_{u v}\left(t_{u}\right)\right)+e_{i}$,

where now the $\Gamma$ functions denote the probability of having a QTL allele from the $\mathrm{F}_{1}$ parent at position $T$ given the information available. If for a given individual $i$, a particular position $T$ is flanked by two markers (option (ii), above), then $\Gamma_{u}\left(t_{u}\right)=0$ and $\Gamma_{u v}\left(t_{u}\right)$ takes a value defined by:

$$
\begin{aligned}
& \Gamma_{u v}\left(t_{u}\right)=1-\frac{t_{u}\left(\delta_{u}-t_{u}\right)}{\left(1-\delta_{u}\right)\left(1-2 t_{u}\right)} \text { if } L_{u}=M \text { and } L_{v}=M, \\
& \Gamma_{u v}\left(t_{u}\right)=\frac{\left(1-t_{u}\right)\left(\delta_{u}-t\right)}{\delta_{u}\left(1-2 t_{u}\right)} \quad \text { if } L_{u}=M \text { and } L_{v}=m, \\
& \Gamma_{u v}\left(t_{u}\right)=1-\frac{\left(1-t_{u}\right)\left(\delta_{u}-t\right)}{\delta_{u}\left(1-2 t_{u}\right)} \text { if } L_{u}=m \text { and } L_{v}=M, \\
& \Gamma_{u v}\left(t_{u}\right)=\frac{t_{u}\left(\delta_{u}-t_{u}\right)}{\left(1-\delta_{u}\right)\left(1-2 t_{u}\right)} \text { if } L_{u}=m \text { and } L_{v}=m .
\end{aligned}
$$

These functions, $\Gamma_{u v}\left(t_{u}\right)$, assuming no interference, are defined as in eqn 3 but the relevant recombination 
probabilities are now $\delta_{u}$ and $t_{u}$. If in the particular individual $i$, the position $T$ is flanked only by one marker (option (i), above), then $\Gamma_{u v}\left(t_{u}\right)=0$ and $\Gamma_{u}\left(t_{u}\right)$ takes the value

$$
\begin{aligned}
& \Gamma_{u}\left(t_{u}\right)=1-t_{u} \text { if } L_{u}=M, \text { or } \\
& \Gamma_{u}\left(t_{u}\right)=t_{u} \text { if } L_{u}=m ;
\end{aligned}
$$

these are the probabilities of having a QTL allele ' $A$ ' from the $F_{1}$ parent when the marker genotype of locus $u$ is $M$ or $m$.

To estimate the position of the QTL we look for the value of $T, T_{\min }$, that minimizes the Residual Sum of Squares of the model. The estimation of the size of its effect is then given by the least squares estimator of $\beta_{1}$, when fitting eqn 4 at $T_{\min }$.

It is clear that not all the missing information is recovered with this procedure. This is because even when the individuals with missing marker genotypes are included in the analysis, the uncertainty about their missing marker genotype diminishes the quantity of information that these individuals would have contributed if there had been no missing markers.

As we shall see from examples, the recovery of information can often be essential in the interpretation of the analysis.

\section{Analyses of simulated data}

To test the method for recovering information from missing markers, a data set was simulated. The genetical map of the loci was

$\begin{array}{lllllll}L_{1} & L_{2} & L_{3} & Q & L_{4} & L_{5} & L_{6}\end{array}$

where $L_{i}, i=1,2, \ldots 6$ represent marker loci, and ' $Q$ ' denotes a QTL. The distance between each pair of successive markers was $20 \mathrm{cM}$ and the QTL ' $Q$ ' is half way between $L_{3}$ and $L_{4}$. No interference was assumed and two hundred observations arising from the backcross

$$
\begin{aligned}
& M_{1} M_{2} M_{3} A M_{4} M_{5} M_{6} / m_{1} m_{2} m_{3} a m_{4} m_{5} m_{6} \times \\
& m_{1} m_{2} m_{3} a m_{4} m_{5} m_{6} / m_{1} m_{2} m_{3} a m_{4} m_{5} m_{6}
\end{aligned}
$$

were simulated. The environmental error was simulated to have a normal distribution with mean zero and a standard deviation of one. The size of the QTL was set to one standard deviation. The data set with all the observations is referred to here as the complete data set.

To obtain a data set with missing observations, 50 observations ( 25 per cent) were deleted at each marker locus. Under the hypothesis of independent and random missing values, a proportion of $0.25 \times 0.25=0.625$ of the observations will have a given pair of successive markers missing and
$0.25 \times 0.25 \times 0.25=0.0156$ three successive markers missing. The expected numbers of observations with zero, one, two and three successive markers were approximately adjusted to their expected values, so that we have 50 individuals with each particular marker missing; for each pair of successive markers 12 individuals with both markers missing, and for each three successive markers three individuals with the three successive markers missing. Thus the proportion of observations with no missing observations in each pair of markers was 56 per cent, i.e. only 112 observations of the total of 200 were usable in each interval if the method of recovering information is not applied. The marker data to be deleted were selected at random, with the only constraint of fulfilling the expected number of missing values, as stated above. The data set is referred to as the missing data set.

Three analyses were performed. Firstly, the regression mapping model was fitted to each pair of successive markers in the complete data set. Secondly, the same model was fitted to each pair of successive markers in the missing data set, ignoring in each interval observations with one or two missing markers for that interval, i.e. ignoring 56 per cent of the phenotypic information. Thirdly, the algorithm to rescue information from missing data was applied to the missing data set, fitting model 4 . In each case we present a graph of the mean square error of the model,

$\operatorname{MSE}(t)=\operatorname{RSS}(t) /\left(n_{\mathrm{r}}-3\right)$,

where $\operatorname{RSS}(t)$ is the residual sum of squares of the fitted model at position $t, 0 \leq t \leq \delta$ in each interval. The $\operatorname{MSE}(t)$ has $\left(n_{r}-3\right)$ degrees of freedom, where $n_{r}$ is the number of observations taken into account in each analysis in the $r$ th interval, and the three degrees of freedom are subtracted because we are estimating, in each case, three parameters, $\beta_{0}, \beta_{1}$ and $\theta$. When there are no missing values, the criterion of taking the value of $t$ that minimizes the MSE $(t)$ over the whole chromosome is equivalent to taking the value of $t$ that minimizes the $\operatorname{RSS}(t)$ of the model, because we have the same number of degrees of freedom for $\operatorname{MSE}(t)$ in each interval. However, if there are missing values, the number of degrees of freedom may be different for each interval, and thus the $\operatorname{RSS}(t)$ and $\operatorname{MSE}(t)$ values may give conflicting information about the interval in which QTLs are likely to be found. Here we analyse the $\operatorname{MSE}(t)$ for each point of the chromosome. This is because, under the hypothesis of one QTL segregating within the interval studied, $\operatorname{MSE}(t)$ is an estimate of the error variance and so a more uniform criterion to compare neighbouring intervals.

The $F$ value of the analysis of variance of the model can be used to evaluate the significance of the size of the effect of the fitted QTL. Because we have six 
markers we will be doing five tests, one in each interval. Although the five tests are not statistically independent, an approximate adjustment for the multiple testing would be to use a significance level of $0.05 / 5=0.01$ to achieve an overall significance level of about 0.05 in each test. The critical value of the $F$ distribution is then 6.63 , for 1 and $n_{r}-3$ degrees of freedom, provided that $n_{r}$ is large.

Figure 1 presents the results of the analysis for the complete data set. This figure shows the graph of $\operatorname{MSE}(t)$ for each point of the chromosome (distances are in centiMorgans in the graph). From this graph we can see that the global minimum of the $\operatorname{MSE}(t)$ is located between the marker loci $L_{3}$ and $L_{4}$, at $48 \mathrm{cM}$ from $L_{1}$. This minimum signals the presence of a QTL at that position, with estimated size of effect $\hat{\beta}_{1}=1.21$ and an estimated standard error for this size of effect of 0.16 . The $F$ value for the analysis of variance of the fitted model at that point is equal to 60.25 , which is highly significant. In this graph we can observe another minimum of the $\operatorname{MSE}(t)$, at approximately $36 \mathrm{cM}$ from $L_{1}$, between markers $L_{2}$ and $L_{3}$. This minimum is a reflected effect of the QTL, present between $L_{3}$ and $L_{4}$. Three marker regression mapping, using markers $L_{2}, L_{3}$ and $L_{4}$, and fitting two QTLs at the same time could be used to test the hypothesis of another QTL between markers $L_{2}$ and $L_{3}$. From such an analysis we would conclude, correctly, that there is only one QTL, with estimated position $48 \mathrm{cM}$ from $L_{1}$ and an estimated size of effect of 1.21 .

Figure 2 presents the results of the analysis of the missing data set, ignoring in each interval any observa-

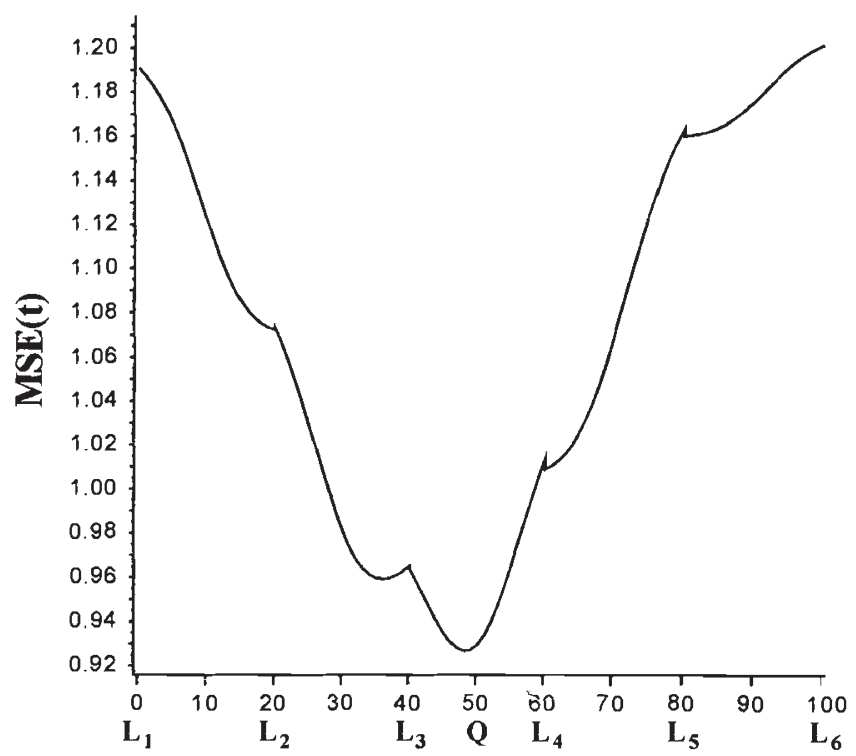

Fig. 1 Regression mapping analysis of the complete simulated data set. Mean square error, $\operatorname{MSE}(t)$, for each point of the chromosome between marker loci $L_{1}$ and $L_{6}$. tion with one or more markers missing, and fitting model 2. This graph shows the $\operatorname{MSE}(t)$ for each point of the chromosome between $L_{1}$ and $L_{6}$. This graph presents very long gaps at the positions of each one of the marker loci $L_{2}$ to $L_{5}$. The gaps are, as mentioned before, the result of fitting model 2 to different sets of observations, given that the individuals with missing

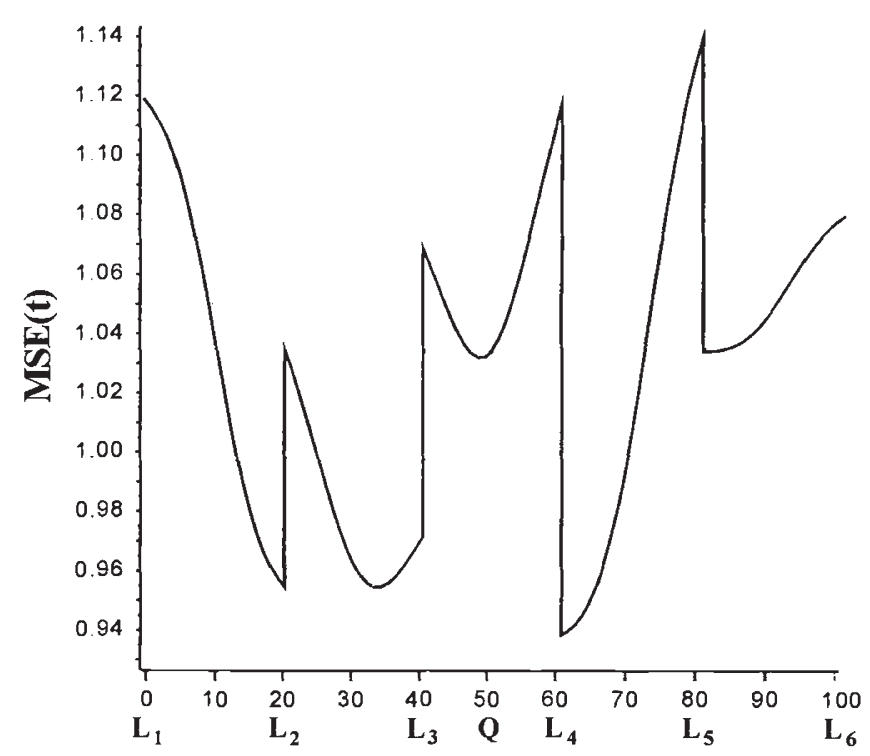

Fig. 2 Regression mapping analysis of the missing simulated data set when ignoring individuals with missing markers in each interval. Mean square error, $\operatorname{MSE}(t)$, for each point of the chromosome between marker loci $L_{1}$ and $L_{6}$.

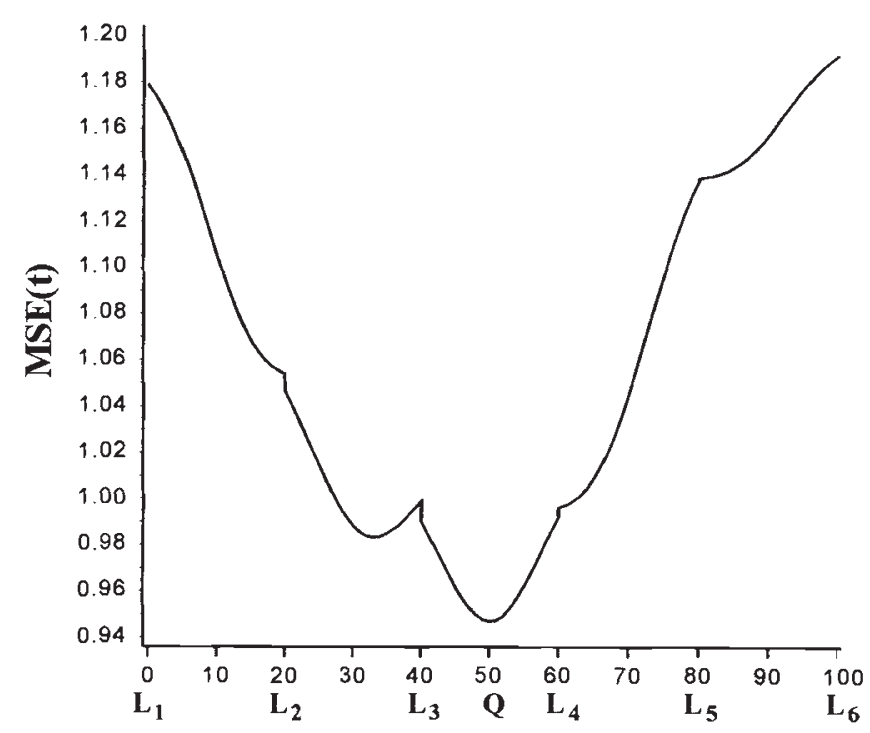

Fig. 3 Regression mapping analysis of the missing simulated data set when using the method to recover information from individuals with missing markers in each interval. Mean square error, $\operatorname{MSE}(t)$, for each point of the chromosome between marker loci $L_{1}$ and $L_{6}$. 
observations for, say markers $L_{1}$ and $L_{2}$, are different from the individuals with missing observations for marker loci $L_{2}$ and $L_{3}$. In this case the number of missing observations is the same for each interval, because we used the expected number of missing values under the assumption of missing values occurring independently. Consequently we have the same numbers of degrees of freedom for the $\operatorname{MSE}(t)$ in each interval, and so the gaps at each of the marker loci are the result only of fitting a partially different data set in each interval. The gaps in the $\operatorname{MSE}(t)$ function make it very difficult to interpret the results of the analysis. Note that the global minimum of the $\operatorname{MSE}(t)$ is reached at marker locus $L_{4}$. The graphs of the MSE $(t)$ in intervals $L_{2}-L_{3}$ and $L_{3}-L_{4}$ both show a minimum between the markers, making it impossible to decide, without an analysis of three marker loci at a time, if there is a QTL between $L_{2}-L_{3}$ or $L_{3}-L_{4}$. The maximum $F$ value for the analysis of the missing data set, ignoring missing observations is located at marker $L_{4}$, with a value of 32; again highly significant.

Figure 3 presents the results from the analysis of the missing data set when using the method to recover the information from individuals with one or more missing observations, i.e. fitting model 4 to the missing data set. Figure 3 shows the $\operatorname{MSE}(t)$ for the model 4, fitted to the missing data set, in each interval. The graph presents small gaps at the marker loci $L_{2}, L_{3}$ and $L_{4}$. These small gaps are the result of using different pairs of markers on the two sides of each marker locus, $L_{1}$, $L_{2}, L_{3}$ and $L_{4}$, to classify the individuals into the four marker classes. However, the results from successive intervals are comparable. A global minimum of the $\operatorname{MSE}(t)$ is present between markers $L_{3}$ and $L_{4}$, at 50 cM from $L_{1}$. This minimum signals the presence of a QTL $Q$ at that point. The estimated size of the effect for the QTL at that position is $\hat{\beta}_{1}=1.23$, with an estimated standard error for the size of effect of 0.17 and an $F$ value for the model at this point of 54.86. As in the case of the analysis of the complete data set, this value is highly significant. The estimated standard error of $\hat{\beta}_{1}$ compares well with the standard error of $\hat{\beta}_{1}$ when the complete data set is analysed. As in the case of Fig. 1 , another minimum of the $\operatorname{MSE}(t)$ is present in Fig. 3 between markers $L_{2}$ and $L_{3}$ at approximately $33 \mathrm{cM}$ from $L_{1}$. This minimum is the result of the effect of QTL $Q$, reflected in the interval $L_{2}-L_{3}$. As said before, the presence of a QTL in the interval $L_{2}-L_{3}$ can be tested using three markers regression mapping with markers $L_{2}, L_{3}$ and $L_{4}$. Later we discuss the recovery of information from missing markers when using three markers at the same time.

Comparing Figs 1 and 3, we can see that the method to recover information from the missing values has been very efficient. The global minimum of the $\operatorname{MSE}(t)$ is located approximately in the same place in both figures, between markers $L_{3}$ and $L_{4}$, giving in both cases a good estimate of the position of the QTL. Both analyses give similar estimates of the size of the QTL effect: 1.21 and 1.23 , respectively.

\section{Analysis of a barley data set}

The data set consists of observations of six molecular markers (denoted here as $L_{1}, L_{2}, \ldots L_{6}$ ) and a quantitative character (mildew resistance) in 112 double haploid lines of barley. The map of the six loci, located on chromosome one, is presented in Figs 4 and 5. In both cases the units are Kosambi centiMorgans, measured from $L_{1}$. The percentage of missing observations from each marker locus was 14.3 per cent for $L_{1}$, 2.7 per cent for $L_{2}, 1.8$ per cent for $L_{3}$ and $L_{4}, 5.4$ per cent for $L_{5}$ and 50 per cent for $L_{6}$. As seen before, a particular observation is missing for a given interval if one or both markers are missing. The percentages of missing observations for each interval were 17 per cent for $L_{1}-L_{2}, 4.5$ per cent for $L_{2}-L_{3}, 2.7$ per cent for $L_{3}-L_{4}, 5.4$ per cent for $L_{4}-L_{5}$ and 53.6 per cent for $L_{5}-L_{6}$.

Double haploid lines are a random sample of the $F_{1}$ gametes, and thus the genetic background of the problem is equivalent to a backcross, with the advantage that the homozygosity of the individuals is guaranteed. Both types of analysis were applied to the data set; firstly, ignoring the missing observations in each interval and secondly using the method to recover information from missing markers.

Figure 4 presents the results of the analysis of the barley data set where the lines with one or more missing markers in each interval were ignored. This figure presents the graph of the $\operatorname{MSE}(t)$ resulting from fitting model 2 to the data set with missing observations for each point in the chromosome between marker loci $L_{1}$ and $L_{6}$. This graph presents gaps at each marker locus, resulting from the different set of missing observations for each interval.

The global minimum of the $\operatorname{MSE}(t)$ in Fig. 4 is located at locus $L_{5}$. At this position, and using the information from loci $L_{5}$ and $L_{6}$, the estimated size of the QTL is 0.80 , with a standard error of 0.21 . The estimate of the error variance at this point, given by the $\operatorname{MSE}(t)$, has a value of 0.66 and the corresponding $F$ value of the analysis of variance is equal to 12 , with 1 and 49 degrees of freedom. This $F$ value is significant at 0.1 per cent. If we take the estimate of the size of effect at locus $L_{5}$, but using the information from marker loci $L_{4}$ and $L_{5}$, the estimated size of effect is equal to 0.63 , with a standard error of 0.14 . The esti- 


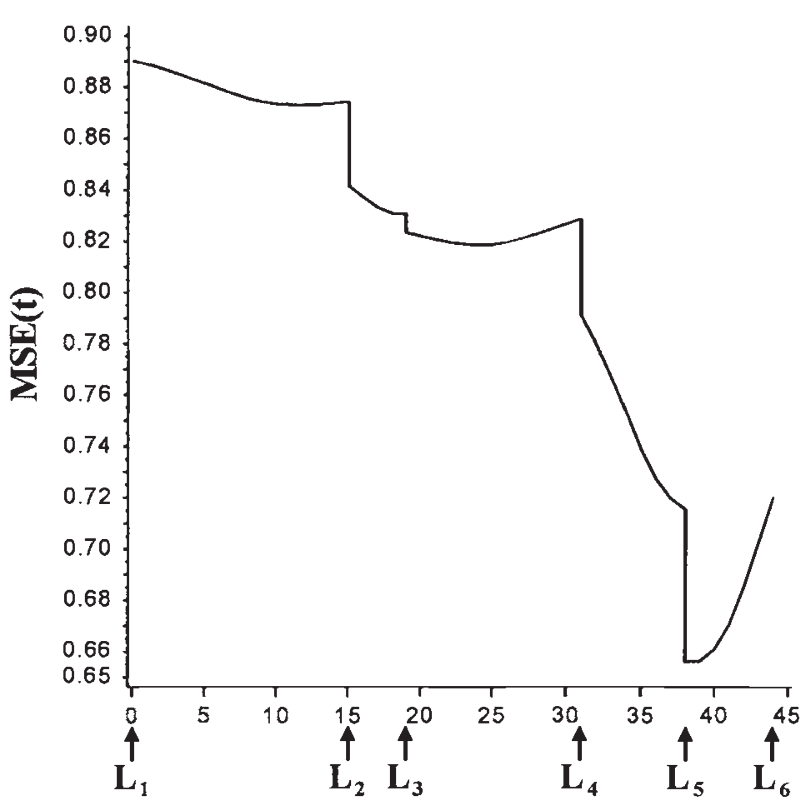

Fig. 4 Regression mapping analysis of the barley data set when individuals with a missing marker in each interval are ignored in the analysis. Mean square error, $\operatorname{MSE}(t)$, for each point of the chromosome between marker loci $L_{1}$ and $L_{6}$.

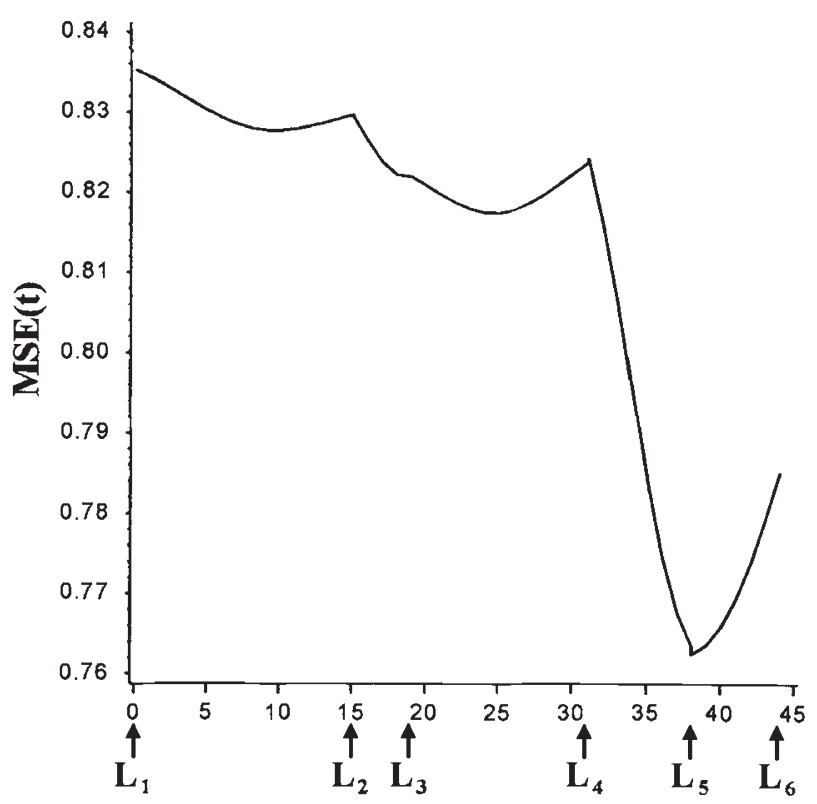

Fig. 5 Regression mapping analysis of the barley data set when using the method to recover information from individuals with missing markers in each interval. Mean square error, $\operatorname{MSE}(t)$, for each point of the chromosome between marker loci $L_{1}$ and $L_{6}$.

mated error variance is in this case 0.72 , and the corresponding $F$ value of the analysis of variance is equal to 15 , with 1 and 103 degrees of freedom. This value is also significant at 0.1 per cent.
As seen from the previous paragraph, two different estimates of the size of the QTL effect, 0.80 and 0.63 , are obtained when using different marker pairs, $L_{5}-L_{6}$ and $L_{4}-L_{5}$ respectively, at the same point of the chromosome, $L_{5}$. This difference in the estimates is a result of using sets of data that have different missing observations. Also the $\mathrm{F}$ values obtained at $L_{5}$ using marker pairs $L_{5}-L_{6}$ and $L_{4}-L_{5}$ are not directly comparable, because we have different numbers of missing observations for each interval and thus different numbers of degrees of freedom for the denominator of these $F$ values.

Figure 5 presents the results of the analysis of the barley data set when the method to recover information from missing markers is applied. In Fig. 5 the graph of the $\operatorname{MSE}(t)$ for each point between the markers $L_{1}$ and $L_{5}$ has no visible gaps at the marker loci. The global minimum of the $\operatorname{MSE}(t)$ is, as in Fig. 4, located at marker locus $L_{5}$ but in contrast with Fig. 4 the value of the $\operatorname{MSE}(t)$ at $L_{5}$ is almost the same when using information from the marker pairs $L_{4}-L_{5}$ or $L_{5}-L_{6}$. The two values differ only because some of the individual plants are classified in different marker classes when using information from markers $L_{4}$ and $L_{5}$ than when using information from markers $L_{5}$ and $L_{6}$. Assuming the QTL is exactly at locus $L_{5}$ the estimated value of the size of the QTL effect is 0.56 , with standard error of 0.17 . At this point the estimated error variance, given by $\operatorname{MSE}(t)$, is equal to 0.76 and the corresponding $F$ value from the analysis of variance is 11.6, with 1 and 109 degrees of freedom. This value is significant at 0.1 per cent.

Comparing Figs 4 and 5 we can see how the recovery of missing information virtually eliminates the gaps present at marker positions when individuals with missing observations are ignored. From these figures we can also note a decrease in the range of $\operatorname{MSE}(t)$ values in Fig. 5 compared with the range of $\operatorname{MSE}(t)$ values in Fig. 4. This indicates that taking all the available information into account increases the precision of the estimate of the error variance, given by the $\operatorname{MSE}(t)$. Another difference between the analyses is the estimated size of the QTL effect.

\section{Generalizing the method}

Regression mapping models can use the information of three or more successive marker loci to estimate two or more QTLs segregating in neighbouring intervals (Haley \& Knott, 1992; Martínez \& Curnow, 1992). As in the case of two markers regression mapping, we can use information from auxiliary non missing markers to recover partially the information from missing markers. The generalization of the method of 
recovering information to other regression mapping models in other segregating populations is straightforward. As in the case presented here of two marker regression mapping in backcross populations, we need to recalculate the probabilities of having specific QTL genotypes at putative positions on the chromosome using the markers available in each individual and use these putative probabilities in the regression of phenotypic values of the character.

\section{Discussion}

When there are missing data and two marker regression mapping models are applied to successive intervals, the resulting $\operatorname{RSS}(t)$ or MSE $(t)$ graphs can present large gaps at each marker position. These gaps are the consequence of: (i) small random variations in the RSS given by the division of the individuals into four groups instead of two, (ii) different individuals having different missing markers for successive pairs of markers, and (iii) the number of missing observations being different for each pair of markers. The algorithms presented here diminish the size of the gaps by recovering information and thus diminishing the importance of (ii) and practically eliminating (iii). When interpreting the $\operatorname{MSE}(t)$ graphs we are looking for clear minima, that will signal the presence of QTLs in the chromosome. Even when no missing values exist, the interpretation of these graphs is complicated by the fact that the effect of one QTL can be reflected in neighbouring intervals, giving a local minimum where no QTL exists. Normally, the minimum in the interval where the QTL is present will be the global minimum of the graph, pointing correctly at the approximate location of the gene. Reflected effects of the QTL in neighbouring intervals can be tested and discarded as real QTLs by the use of three markers regression mapping (Haley \& Knott, 1992; Martínez \& Curnow, 1992). However, when missing data are present, one of the reflected effects of the QTL may be the global minimum of the $\operatorname{MSE}(t)$, as happened in the simulated data set, where the minima in the intervals $L_{2}-L_{3}$ and $L_{4}-L_{5}$ have smaller values than the minimum in the interval $L_{3}-L_{4}$ where the QTL is actually located (Fig. 2). The presence of missing data may, in some cases, make it impossible to discriminate between the minimum $\operatorname{MSE}(t)$ at which the QTL is located and other minima resulting from the reflected effect of this gene.

As mentioned before, using the recovery method implies a loss of information with respect to the hypothetical complete data set. This is because when we are using auxiliary markers, the recombination distance between them is necessarily larger than the distance between the original markers. Larger recom- bination distance means more genetical variability within the marker groups and this increases the unexplained error variation, that in turn increases the uncertainty about the position of the QTL. However, if the auxiliary markers are not far apart and the proportion of missing markers is less than, say, 25 per cent at each marker locus, the method to recover information appears to be very efficient.

When multiple minima are present in the $\operatorname{MSE}(t)$ graph it is necessary to apply three markers regression mapping models, either to estimate correctly the positions and sizes of two QTLs in neighbouring intervals, or to discard one of the minima as a reflected effect of a real QTL. In this context, the recovery of information from missing markers is essential to the fitting of three markers regression mapping models. This is because, when taking three successive markers into account, the number of missing observations will be larger than the number of missing observations when taking only two markers at a time. For example, if the missing observations occur independently and at random, and if on average 25 per cent of the observations at a marker locus are missing, the percentage of missing observations for two markers will be, on average, 43.75 per cent, while with three markers the percentage of missing values will rise to an average of 57.81 per cent.

We have developed sas programs to perform regression mapping using two or three markers at the same time that include the recovery of information from missing markers. These programs are available on request.

\section{Acknowledgements}

We are grateful to Dr C. J. Skidmore and Mrs S. Hodgson of the Department of Biochemistry and Physiology at the University of Reading and Dr J. Chojecki of Zeneca Seeds for allowing us to include the analysis of some of their data on barley to illustrate the use of our method.

\section{References}

CARBONELL, E. A., GERIG, T. M. AND BALANSARD, E. 1992. Interval mapping in the analysis of nonadditive quantitative trait loci. Biometrics, 48, 305-315.

HALEY, C. S. AND KNOTT, S. A. 1992. A simple regression method for mapping quantitative trait loci in line crosses using flanking markers. Heredity, 69, 315-324.

JANSEN, R. C. 1992. A general mixture model for mapping quantitative trait loci by using molecular markers. Theor. Appl. Genet., 85, 252-260.

KNAPP, S. J., BRIDGES, W. C. AND BIRKES, D. 1990. Mapping quantitative trait loci using molecular marker linkage maps. Theor. Appl. Genet., 79, 583-592. 
LANDER, E. S. AND BotSTEIN, D. 1989. Mapping Mendelian factors underlying quantitative traits using RFLP linkage maps. Genetics, 121, 185-199.

LUO, Z. W. AND KEARSEY, M. J. 1989. Maximum likelihood estimation of linkage between a marker gene and a quantitative locus. Heredity, 63, 401-408.

LUO, Z. W. AND KEARSEY, M. J. 1991. Maximum likelihood estimation of linkage between a marker gene and a quantitative trait locus. II. Application to backcross and doubled haploid populations. Heredity, 66, 117-124.

MARTINEZ, O. AND CURNOW, R. N. 1992. Estimating the locations and the sizes of the effects of quantitative trait loci using flanking markers. Theor. Appl. Genet., 85, 480-488.
SIMPSON, S. P. 1989. Detection of linkage between quantitative trait loci and restriction fragment length polymorphisms using inbred lines. Theor. Appl. Genet., 77, 815-819.

WELleR, J. I. 1989. Maximum likelihood techniques for the mapping and analysis of quantitative trait loci with the aid of genetic markers. Biometrics, 42, 627-640.

WELLER, J. 1. 1987. Mapping and analysis of quantitative trait loci in Lycopersicon (tomato) with the aid of genetic markers using approximate maximum likelihood methods. Heredity, 59, 413-421. 